\title{
Hydrogen Accumulation and Distribution during the Saturation of a VT1-0 Titanium Alloy by an Electrolytic Method and from a Gas Atmosphere
}

\author{
V. N. Kudiyarov*, A. M. Lider, N. S. Pushilina, and N. A. Timchenko \\ National Research Tomsk Polytechnical University, ul. Lenina 30, Tomsk, 634050 Russia \\ *e-mail: viktor.kudiiarov@gmail.com
}

\begin{abstract}
The accumulation, distribution, and thermally stimulated release of hydrogen in a VT1-0 titanium alloy during electrolytic saturation and gas-phase saturation are studied. After electrolytic saturation, a $0.4-\mu \mathrm{m}$-thick surface layer consisting of $\delta$ hydrides with a binding energy of $108 \mathrm{~kJ} / \mathrm{mol}$ forms in the alloy. The hydride dissociation after electrolytic saturation in heating occurs in the temperature range $320-370^{\circ} \mathrm{C}$. After saturation from a gas atmosphere, $\delta$ hydrides with a binding energy of $102 \mathrm{~kJ} / \mathrm{mol}$ form throughout the alloy volume. The dissociation of the hydrides formed during gas-phase saturation in heating occurs in the temperature range $520-530^{\circ} \mathrm{C}$. A further increase in the temperature is accompanied by the transformation of titanium from the $\alpha$ into the $\beta$ modification. At $690-720^{\circ} \mathrm{C}$, the phase transformation is completed, and another hydrogen desorption peak appears in a thermally stimulated hydrogen desorption spectrum.
\end{abstract}

\section{INTRODUCTION}

Titanium and its alloys are widely used as structural materials in various industries $[1-5]$. The penetration and accumulation of hydrogen in titanium parts change their physicochemical and service properties and cause their delayed fracture because of hydrogen embrittlement [6]. The kinetics and intensity of such changes depend on the state of hydrogen in a material [7]. Hydrogen in titanium can be in the form of hydrides or in a dissolved state $[8,9]$. To determine the quantity, distribution, and state of accumulated hydrogen at various types of treatment is necessary to develop methods for preventing hydrogen embrittlement.

To determine the state and quantity of hydrogen accumulated in metals, researchers apply thermodesorption spectroscopy (TDS), where the flow of desorbed hydrogen is continuously measured in heating at a given rate $[10,11]$. In the TDS studies of hydrogen accumulation in titanium alloys, it is important to take into account the effect of the phase transitions in the titanium-hydrogen system on thermally stimulated hydrogen desorption, since they affect the shape of TDS spectra [8, 12]. The hydrogen accumulation in titanium was analyzed by TDS in many works [8-12]. For example, the effect of the phase transitions in the titanium-hydrogen system on the thermally stimulated hydrogen desorption after electrolytic hydrogen saturation was comprehensively studied in [12]. The authors of $[9,10]$ investigated the hydrogen accumulation in titanium by TDS during saturation from a gas atmosphere; however, they did not analyze the effect of the phase transitions in the titanium-hydrogen system on thermally stimulated hydrogen desorption after saturation from a gas atmosphere.

To understand the processes of hydrogen accumulation in titanium alloys, one has to know detailed information on the phase transitions in the titaniumhydrogen system during simultaneous thermal action and hydrogen desorption. Such information can be obtained from the diffraction data measured during TDS experiments using synchrotron radiation. The purpose of this work is to study the hydrogen accumulation and distribution in a commercial-purity titanium alloy (VT1-0) during electrolytic saturation and saturation from a gas atmosphere and to perform in situ investigations of the effect of the phase transitions in the titanium-hydrogen system on the thermally stimulated hydrogen desorption using synchrotron radiation.

\section{EXPERIMENTAL}

For investigations, we prepared $20 \times 20 \times 1-\mathrm{mm}$ samples. They were spark-cut from a VT1-0 titanium alloy sheet in the as-delivered state. The sample surfaces were mechanically polished to remove a surface oxide film. The samples were vacuum annealed at $750^{\circ} \mathrm{C}$ for $60 \mathrm{~min}$ to remove defects and to relieve surface stresses.

Electrolytic saturation was performed in an Msolution of sulfuric acid for $360 \mathrm{~min}$ at an electric current of $0.73 \mathrm{~A}$ and an electrolyte temperature of $70^{\circ} \mathrm{C}$. Saturation from a gas atmosphere was carried out at a 


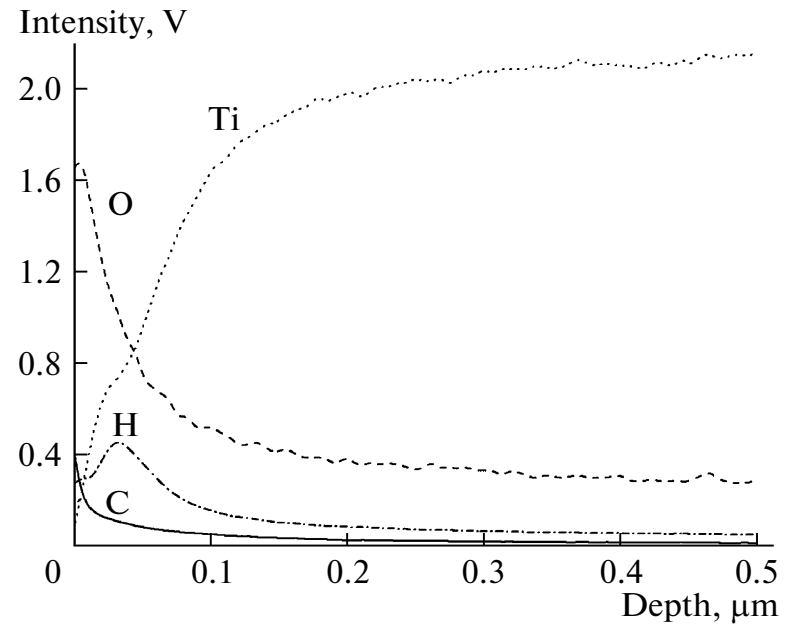

Fig. 1. Depth profiles of the elements in the titanium alloy before hydrogen saturation.

temperature of $600^{\circ} \mathrm{C}$ for $60 \mathrm{~min}$ at a hydrogen pressure of $6.6 \times 10^{4} \mathrm{~Pa}$ in the chamber of a computerassisted Gas Reaction Controller LPB setup [13]. After hydrogen saturation, we measured the hydrogen concentration with an RHEN 602 (LECO) hydrogen analyzer. The depth profiles of hydrogen were recorded using a GD-Profiler 2 (Horiba) glow discharge plasma spectrometer [14]. The phase composition and the structure parameters of the samples after saturation were studied on an XRD-6000 diffractometer using $\mathrm{Cu} K_{\alpha}$ radiation. The phase composition was analyzed using the PCPDFWIN and PDF-4+ databases and the POWDER CELL 2.5 full-profile analysis software package.

The spectra of the thermally stimulated hydrogen desorption from the samples were recorded on a computer-assisted Gas Reaction Controller LPB setup using an RGA 100 (Stanford Research Systems) quadrupole mass spectrometer. A sample was placed in a chamber pumped down to a pressure below $10^{-4} \mathrm{~Pa}$ and was then linearly heated. The hydrogen flow was continuously measured with the mass spectrometer. The heating rate of a sample during TDS was 4$8{ }^{\circ} \mathrm{C} / \mathrm{min}$ and the heating range was $30-900^{\circ} \mathrm{C}$. The spectra of thermally stimulated hydrogen release were analyzed by the Kissinger method [15].

The in situ investigation of the phase transitions in the titanium-hydrogen system during thermally stimulated hydrogen release was performed on the Precision X-ray Diffraction II Station of the Institute of Catalysis, Siberian Branch, Russian Academy of Sciences in synchrotron radiation channel 6 of the VEPP-3 electron storage ring [16, 17]. This station is characterized by an OD-3M one-coordinate detector used in the powder diffractometer. The detector consists of a multiwire proportional gas chamber, a detecting unit with a coordinate processor, and a computer [18]. The one-coordinate detectors record scattered

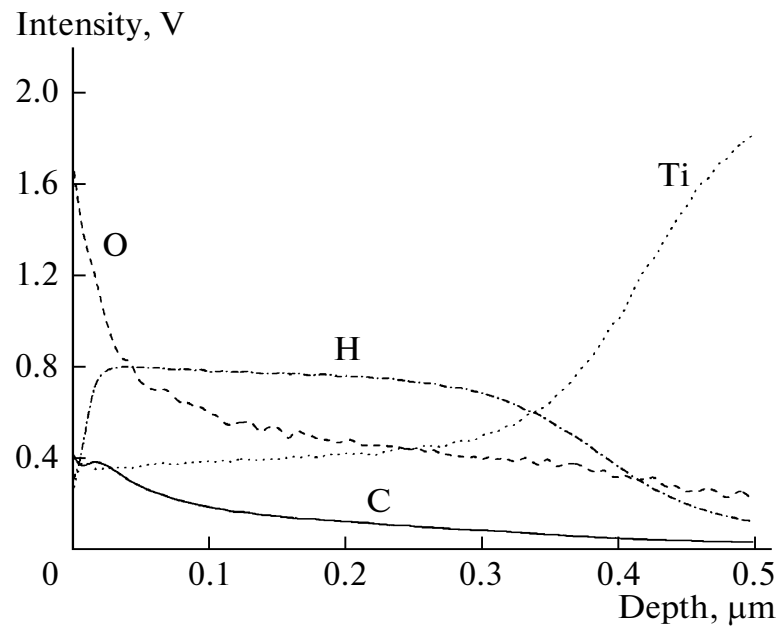

Fig. 2. Depth profiles of the elements in the titanium alloy after electrolytic hydrogen saturation.

radiation in a certain angular range $\left(\sim 30^{\circ}\right)$ in 3328 channels at a speed of response up to $10 \mathrm{MHz}$. A sample was placed in the chamber pumped down to a pressure below $10^{-4} \mathrm{~Pa}$ and was then linearly heated in the range $30-750^{\circ} \mathrm{C}$ at a rate of $6^{\circ} \mathrm{C} / \mathrm{min}$. X-ray diffraction patterns were recorded every heating minute, i.e., every $6^{\circ} \mathrm{C}$. The recorded X-ray diffraction patterns were processed and analyzed using the PDF-2scarch-match, FullProf, and Crystallographica software packages.

\section{RESULTS AND DISCUSSION}

\subsection{Hydrogen Accumulation and Distribution}

Figures 1-3 show the depth profiles of the elements in titanium alloy samples before and after hydrogen saturation. Oxide compounds and gaseous impurities

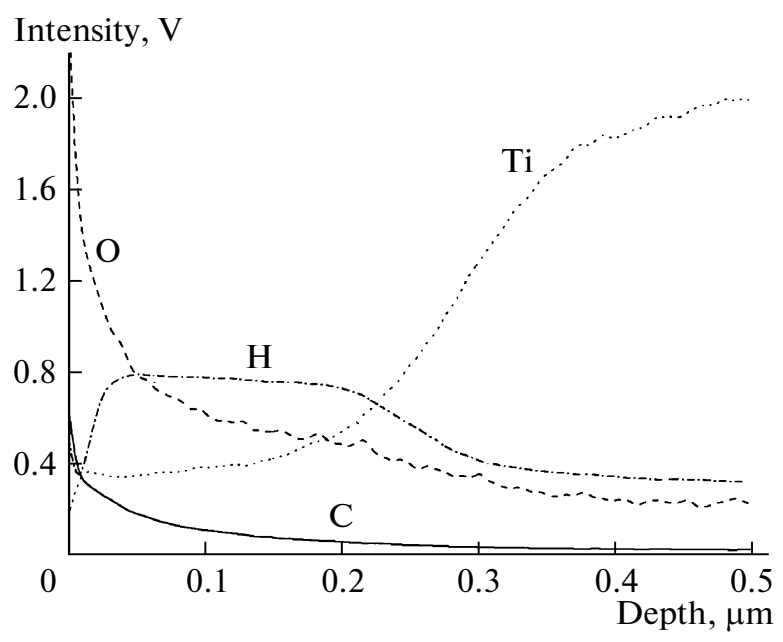

Fig. 3. Depth profiles of the elements in the titanium alloy after gas-phase hydrogen saturation. 
XRD results for the titanium alloy

\begin{tabular}{l|l|c|c}
\hline \multicolumn{1}{c|}{ Samples } & \multicolumn{1}{|c|}{ Detected phases } & Phase content, vol \% & Lattice parameters, $0.1 \mathrm{~nm}$ \\
\hline Initial & Ti_hexagonal & $99.9 \pm 0.1$ & $a=2.941 ; c=4.668$ \\
\hline Electrolytic saturation & Ti_hexagonal & $11.1 \pm 0.1$ & $a=2.917 ; c=4.633$ \\
& $\mathrm{TiH}_{2}$ ccubic & $88.9 \pm 0.6$ & $a=4.3544$ \\
\hline Gas-phase saturation & $\mathrm{Ti}$ hexagonal & $59 \pm 0.5$ & $a=2.936 ; c=4.665$ \\
& $\mathrm{TiH}_{2 \_}$cubic & $40.26 \pm 0.3$ & $a=4.3846$ \\
\hline
\end{tabular}

(carbon, hydrogen) are present in a $0.1-\mu \mathrm{m}$-thick surface layer. In the initial titanium alloy, hydrogen exists in a thin surface region and its absolute content is $0.0034 \mathrm{wt} \%$.

A $0.4-\mu m$-thick layer with a high hydrogen content forms in samples as a result of electrolytic hydrogen saturation (Fig. 2). In this case, the signal intensity of hydrogen is approximately twice as high as the signal intensity of titanium, and an analysis of the depth profile of hydrogen suggests that the layer consists of titanium hydride with a composition close to stoichiometric composition $\mathrm{TiH}_{2}$. The depth profiles of hydrogen in titanium alloy samples after saturation from a gas atmosphere are characterized by the fact that the hydrogen level remains high across the entire analysis depth $(3 \mu \mathrm{m})$, and the hydride layer thickness is $0.25 \mu \mathrm{m}$. This finding is explained by the fact that, during electrolytic saturation, hydrogen accumulates in titanium in the near-surface layer and weakly diffuse deep into the sample volume because of the low diffusion coefficient of hydrogen in titanium at moderate temperatures [7]. During hydrogen saturation from a gas atmosphere at high temperatures, hydrogen diffuses deep into the sample volume and is distributed over the entire sample volume at a sufficient saturation time. The absolute hydrogen content was 0.966 wt \%

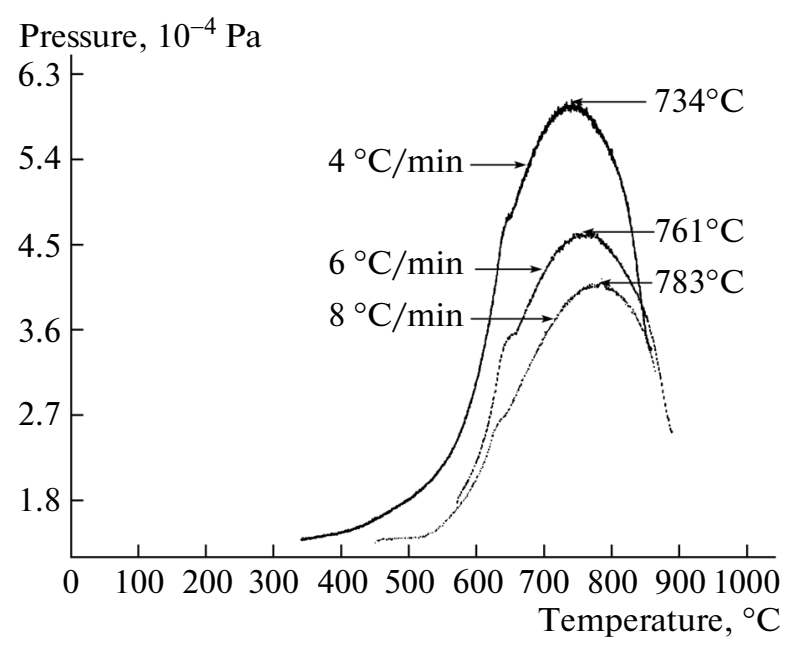

Fig. 4. Spectra of thermally stimulated hydrogen desorption from the titanium alloy after electrolytic saturation. in the samples saturated from a gas atmosphere at $600^{\circ} \mathrm{C}$ and $0.0241 \mathrm{wt} \%$ in the samples subjected to electrolytic saturation.

\subsection{Effect of Hydrogen on the Phase Composition of the Titanium Alloy}

The results of X-ray diffraction (XRD) analysis of the effect of hydrogen saturation on the phase composition of the titanium alloy are given in the table.

During electrolytic saturation, hydrogen accumulates in the near-surface layer of the samples and forms hydride phases, the compositions of which are close to the stoichiometric composition, with the titanium alloy (Fig. 2). This follows from the XRD results (see the table). Hydrogen saturation from a gas atmosphere at a high temperature is accompanied by hydrogen diffusion without the formation of hydride phases [12]. Upon cooling after saturation, hydrides form in the samples; however, the volume content of hydrides on the surfaces of the samples saturated from a gas atmosphere is lower than that of hydrides at the surfaces of the samples subjected to electrolytic saturation (see the table).

\subsection{Thermally Stimulated Hydrogen Desorption}

The spectra of thermally stimulated hydrogen desorption from titanium alloy samples are shown in Fig. 4 (electrolytic saturation) and Fig. 5 (gas-phase saturation). As was detected earlier, the absolute hydrogen content in the samples saturated from a gas atmosphere is higher than in the samples subjected to electrolytic saturation. Thus, the intensity of hydrogen release from the samples saturated from a gas atmosphere (increase in the pressure from $1 \times 10^{-3}$ to $2 \times 10^{-2} \mathrm{~Pa}$ ) is two orders of magnitude higher than that from the samples subjected to electrolytic saturation (increase in the pressure from $1.8 \times 10^{-4}$ to $6.2 \times$ $\left.10^{-4} \mathrm{~Pa}\right)$.

The binding energy of hydrogen for each saturation method was determined from the dependences of $\ln \left(\beta / T^{2}\right)$ on $1 / T$, where $\beta$ is the heating rate and $T$ is the peak temperature (Fig. 6) [10]. As a result, we obtained the following binding energies of hydrogen: $108 \mathrm{~kJ} / \mathrm{mol}$ for electrolytic saturation and $102 \mathrm{~kJ} / \mathrm{mol}$ for gas-phase saturation. These data agree with the 


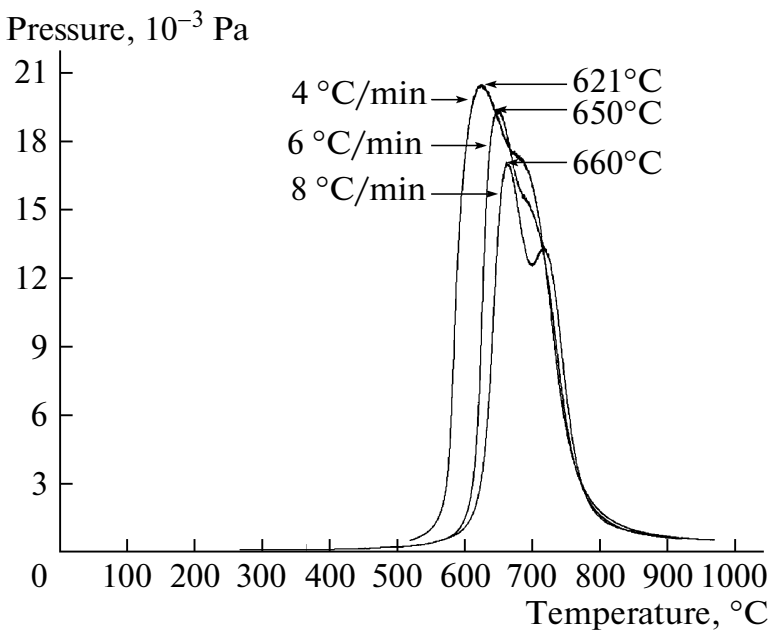

Fig. 5. Spectra of thermally stimulated hydrogen desorption from the titanium alloy after gas-phase saturation.

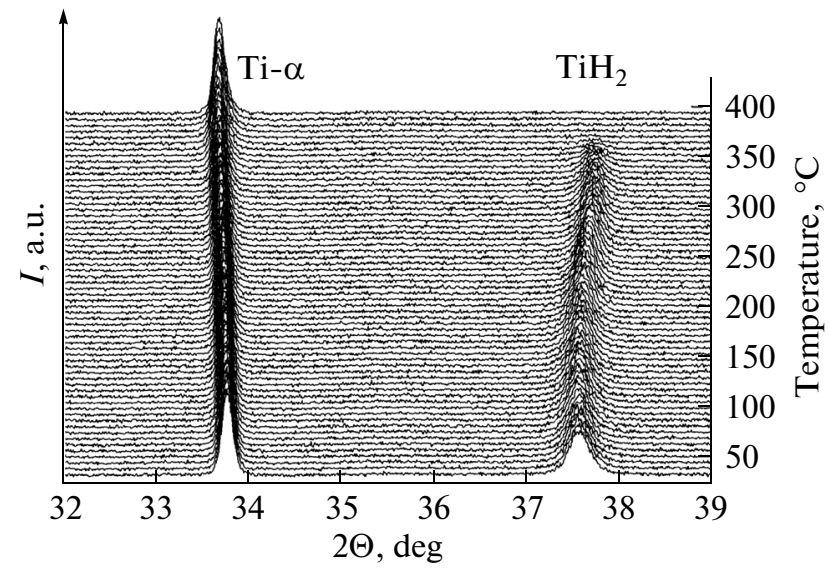

Fig. 7. Kinetics of the phase transitions in heating of the titanium alloy after electrolytic hydrogen saturation.

results $[12,19]$ on determining the binding energy of hydrogen in a titanium alloy after electrolytic saturation $(108$ and $106 \mathrm{~kJ} / \mathrm{mol})$ and gas-phase saturation $(100 \mathrm{~kJ} / \mathrm{mol})$. This binding energy range for hydrogen in titanium corresponds to hydride, which agrees with our XRD data.

The TDS spectra of the samples saturated from a gas atmosphere are characterized by the appearance of a second peak in the high-temperature region, which is related to phase transitions in the titanium-hydrogen system.

\subsection{Phase Transitions in the Titanium-Hydrogen System during Thermally Stimulated Hydrogen Desorption}

Figure 7 shows the X-ray diffraction patterns that demonstrate the kinetics of the phase transitions in annealing of the titanium alloy samples subjected to

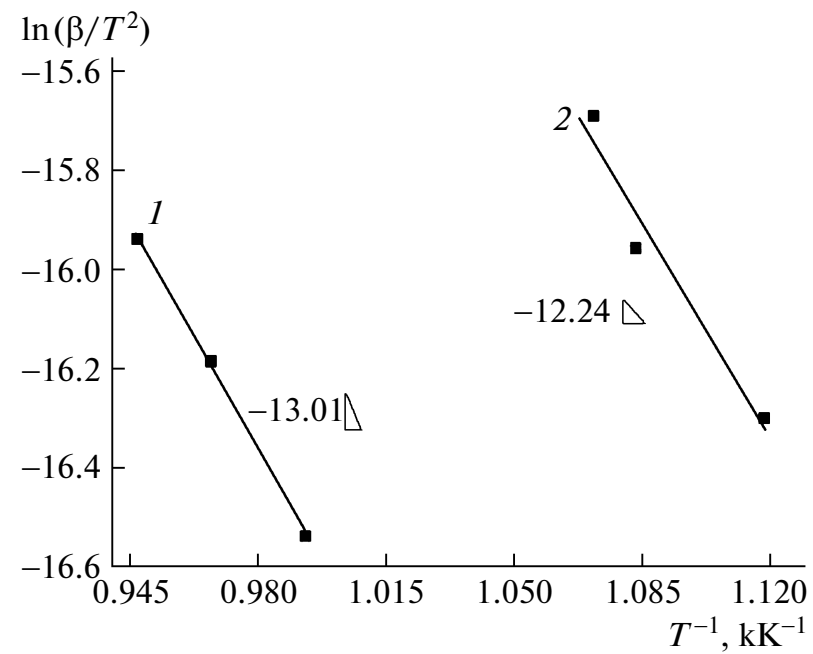

Fig. 6. $\ln \left(\beta / T^{2}\right)$ vs. $1 / T$ for ( 1 ) electrolytic saturation and (2) gas-phase saturation.

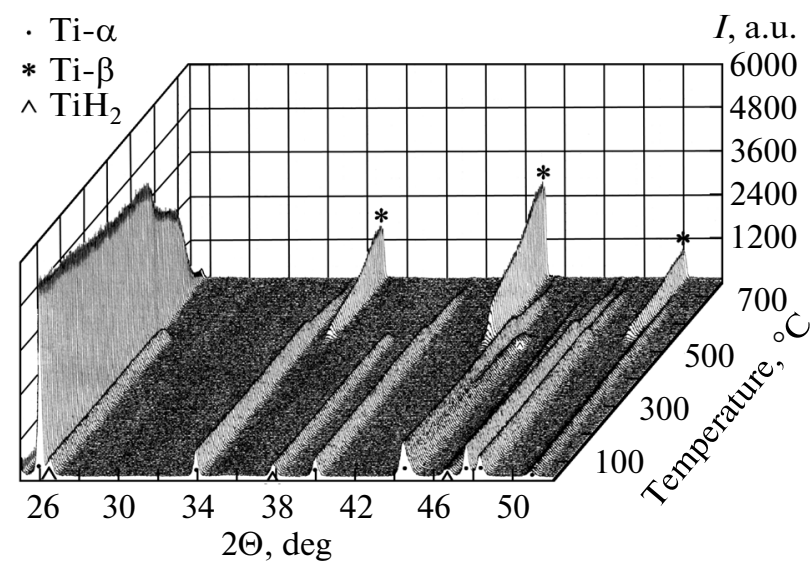

Fig. 8. Kinetics of the phase transitions in heating of the VT1-0 titanium alloy after gas-phase hydrogen saturation.

electrolytic hydrogen saturation. During linear heating at a rate of $6^{\circ} \mathrm{C} / \mathrm{min}$, the reflections of titanium hydride begin to decrease at a temperature of $320^{\circ} \mathrm{C}$ and fully disappear at $370^{\circ} \mathrm{C}$. However, hydrogen desorption begins at a temperature of $550^{\circ} \mathrm{C}$ (Fig. 4). These results demonstrate that hydrogen atoms dissolve in the titanium lattice after dissociation. The hydride dissociation temperature $\left(320^{\circ} \mathrm{C}\right)$ corresponds to the boundary between the $\alpha+\delta$ and $\alpha+\beta$ phase fields in the phase diagram [12]. Thus, the hydrogen electrolytic saturation of the titanium alloy results in the formation of a surface layer consisting of $\delta$ hydrides in the alloys samples.

Figure 8 shows the $\mathrm{X}$-ray diffraction patterns that demonstrate the kinetics of the phase transitions in heating in the titanium alloy samples hydrogen saturated from a gas atmosphere.

During linear heating at a rate of $6{ }^{\circ} \mathrm{C} / \mathrm{min}$, the reflections of titanium hydride begin to decrease at a 
temperature of $520^{\circ} \mathrm{C}$ and fully disappear at $530^{\circ} \mathrm{C}$, and hydrogen desorption begins (Fig. 5). However, hydrogen desorption begins at a temperature of $550^{\circ} \mathrm{C}$ (Fig. 5). The hydride dissociation temperature $\left(530^{\circ} \mathrm{C}\right)$ corresponds to the boundary between the $\delta$ and $\beta+\delta$ phase fields in the phase diagram [12]. Thus, the hydrogen saturation of the titanium alloy from a gas atmosphere results in the formation of $\delta$ hydrides throughout the alloy volume.

A further increase in the temperature from $530^{\circ} \mathrm{C}$ is accompanied by a decrease in the reflection intensities of $\alpha$-titanium and by an increase in the reflection intensities of $\beta$-titanium, which indicates gradual transformation of titanium from the $\alpha$ into the $\beta$ modification. At a temperature of $690-720^{\circ} \mathrm{C}, \alpha$-titanium fully transforms into $\beta$-titanium, and another peak appears in a thermally stimulated hydrogen desorption spectrum (Fig. 5).

\section{CONCLUSIONS}

We experimentally detected that the electrolytic hydrogen saturation of a VT1-0 titanium alloy in an M-solution of sulfuric acid at an electric current of $0.73 \mathrm{~A}$ and an electrolyte temperature of $70^{\circ} \mathrm{C}$ for $360 \mathrm{~min}$ results in the formation of a $0.4-\mu \mathrm{m}$-thick surface layer consisting of $\delta$ hydrides with a binding energy of $108 \mathrm{~kJ} / \mathrm{mol}$. The dissociation of the electrolytically formed hydrides in heating occurs in the temperature range $320-370^{\circ} \mathrm{C}$. The hydrogen saturation from a gas atmosphere at a temperature of $600^{\circ} \mathrm{C}$ for $60 \mathrm{~min}$ and a hydrogen pressure of $6.6 \times 10^{4} \mathrm{~Pa}$ in a chamber results in the formation of $\delta$ hydrides with a binding energy of $102 \mathrm{~kJ} / \mathrm{mol}$ throughout the sample volume. The dissociation of the hydrides formed during gas-phase saturation in heating occurs in the temperature range $520-530^{\circ} \mathrm{C}$. A further increase in the temperature is accompanied by the transformation of titanium from the $\alpha$ into the $\beta$ modification. At $690-$ $720^{\circ} \mathrm{C}, \alpha$-titanium almost fully transformed into $\beta$ titanium, and another hydrogen desorption peak appears in a thermally stimulated hydrogen desorption spectrum.

\section{ACKNOWLEDGMENTS}

I am grateful to A.N. Shmakov for his assistance with this study at the Precision X-ray Diffraction II
Station, Institute of Catalysis, Siberian Branch, Russian Academy of Sciences.

\section{REFERENCES}

1. K. Wang, Mater. Sci. Eng., A 213, 134 (1996).

2. I. Gurrappa, Mater. Characterization 51, 131 (2003).

3. R. W. Schutz and H. B. Watkins, Mater. Sci. Eng., A 243, 305 (1998).

4. M. Yamada, Mater. Sci. Eng., A 213, 8 (1996).

5. W. D. Brewer, R. K. Bird, and T. A. Wallace, Mater. Sci. Eng., A 243, 299 (1998).

6. V. Madina and I. Azkarate, Int. J. Hydrog. Energy 34, 5976 (2009).

7. E. Lunarska, O. Chernyayeva, D. Lisovytskiy, et al., Mater. Sci. Eng., C 30, 181 (2010).

8. Y. Furuya, A. Takasaki, K. Mizuno, et al., J. Alloys Compd. 446-447, 447 (2007).

9. D. Eliezer, E. Tal-Gutelmacher, C. E. Cross, et al., Mater. Sci. Eng. A 421, 200 (2006).

10. E. Tal-Gutelmacher, D. Eliezer, and E. Abramov, Mater. Sci. Eng. A 445-446, 625 (2007).

11. F. Zeppelin, M. Haluska, and M. Hirscher, Thermochim. Acta 404, 251 (2003).

12. A. Takasaki, Y, Furuya, K. Ojima, et al., J. Alloys Compd. 224, 269 (1995).

13. L. V. Gulidova, V. N . Kudiyarov, N. A. Dubrova, and A. M. Lider, Al'tern. Energ. Ekol., No. 03/2 (122), 32 (2013).

14. A. M. Lider, N. S. Pushilina, V. N. Kudiiarov, et al., Appl. Mech. Mater. 302, 92 (2013).

15. S. M. Lee and J. Y. L. Lee, Appl. Phys. 63, 4758 (1988).

16. A. N. Shmakov, B. P. Tolochko, I. L. Zhogin, and M. A. Sheromov, "X-ray synchrotron radiation, neutrons and electrons for investigation of nanosystems and materials," in Proceeding of the 7th National Conference on Nano-Bio-Info-Cognitive Technologies, RSNENBIK, IKRAN-RNTsKI, Moscow, 16-21 November, 2009 , p. 559.

17. A. N. Shmakov, M. G. Ivanov, B. P. Tolochko, M. R. Sharafutdinov, A. I. Ancharov, I. L. Zhogin, and M. A. Sheromov, in Proceedings of the 18th International Conference on the Applications of Synchrotron Radiation, Novosibirsk, 2010, p. 68.

18. V. M. Aul'chenko, Proceedings of the School of Young Specialists on Synchrotron Radiation: Diffraction and Scattering, Novosibirsk, 2009, pp. 6-9.

19. L. Yan, S. Ramamurthy, J. J . Noel, et al., Electrochim. Acta 52, 1169 (2006). 